\title{
Los sistemas de triage: respuesta a la saturación en las salas de urgencias ${ }^{1}$
}

\author{
Consuelo Cubero-Alpízar²
}

Institución: Universidad de Costa Rica

\section{COMO CITAR}

Cubero-Alpízar, C. (2014). Los sistemas de triage: respuesta a la saturación en las salas de urgencias. Rev. Enfermería Actual en Costa Rica, 27, 1-12. DOI: http://dx.doi.org/10.15517/revenf.v0i27.16145

\section{RESUMEN}

Antecedentes. Las estrategias para la atención de usuarios en las salas de urgencias a nivel hospitalario se han vuelto imprescindibles para el buen funcionamiento de estos servicios, debido al incremento de en la demanda cada vez mayor de prestación de servicios en. En este contexto, se plantea un proyecto de investigación cuyo objetivo se centró en analizar la efectividad de los sistemas de atención de urgencias hospitalarias a nivel mundial. Método. Se revisó toda aquella evidencia publicada durante los últimos 15 años, y que incluya observación del personal que los aplica, los tipos de sistema de triage, así como los tiempos de espera antes de la atención y la calidad de la atención brindada al paciente. La estrategia de búsqueda se utilizó para identificar estudios clínicos en diferentes bases de datos como Pubmed, Cochrane library, EBSCO y google académico.

Resultado. Entre los resultados más relevantes se identificó la saturación en las salas de urgencias como un problema real originado del aumento en la demanda de tales servicios a nivel mundial, lo cual redunda en una menor calidad en el servicio que se presta y también en un aumento en la mortalidad por los tiempos de espera. Se identificó que no se compara la atención aleatoria por llegada y los sistemas estructurados, además de que históricamente son las enfermeras las que mejor cumplen dicha tarea.

Conclusión. Es urgente revisar los sistemas de clasificación de pacientes en el momento en el que llegan al área de urgencias del sistema hospitalario, con el objetivo de adaptar los sistemas de triage a las necesidades reales del país.

Palabras clave: Enfermería; saturación-salas-de urgencias-hospitalarias; triage.

\footnotetext{
${ }^{1}$ Fecha de recepción: 9 de mayo del 2014

Fecha de aceptación: 3 de setiembre del 2014

${ }^{2}$ Escuela de Enfermería, Universidad de Costa Rica. Costa Rica. Correo electrónico: ccuberoalpzar@gmail.com
} 


\title{
Triage systems: saturation in response to emergency rooms ${ }^{3}$
}

Consuelo Cubero Alpízar ${ }^{4}$

\section{Institution: University of Costa Rica}

\section{CITED:}

Cubero-Alpízar, C. (2014). Triage systems: saturation in response to emergency rooms. Rev. Enfermería Actual en Costa Rica, 27, 1-12. DOI: http://dx.doi.org/10.15517/revenf.v0i27.16145

\begin{abstract}
Background. Strategies for the attention of users in emergency rooms in hospitals have become indispensable for the proper functioning of these services, due to increase in the growing demand for service delivery. In this context, a research project whose objective was to analyze the effectiveness of care systems worldwide hospital emergency arises.

Method. All that was reviewed evidence published over the past 15 years, including staff observation applies to the system types triage and waiting times before the care and quality of care provided to the patient. The search strategy was used to identify clinical studies in different databases such as Pubmed, Cochrane library, EBSCO and google scholar.

Result. Among the most relevant results identified saturation in emergency rooms as a real problem caused the increase in demand for such services globally, resulting in a lower quality of service provided and also an increase in mortality timeouts. Identified the random arrival care and structured, plus they are historically nurses which systems best meet the task does not compare.
\end{abstract}

Conclusion. It is urgent to review the classification systems of patients when they arrive at the emergency department of the hospital system, with the goal of triage systems adapt to the real needs of the country.

Key words: nursing; saturation-halls-of-hospital-emergency; triage

\footnotetext{
${ }^{3}$ Date of receipt: May 9, 2014

Date of acceptance: September 3, 2014

${ }^{4}$ School of Nursing, University of Costa Rica. Costa Rica. E-mail: ccuberoalpzar@gmail.com
} 


\section{INTRODUCCIÓN}

Los sistemas de atención de urgencias a nivel hospitalario se han visto afectados por un aumento en la demanda de sus servicios a nivel mundial; las causas de este problema son variadas, sin embargo, es claro que esa saturación repercute en una menor calidad en el servicio que se presta y también en un aumento en la mortalidad, asociada directamente con los tiempos de espera (Flores, 2011).

Ante tal problema, pueden implementarse diversas estrategias, las cuales, en su mayoría, reciben la influencia la cultura donde se desarrollan, y toman como base la categorización que tiene como fuente ese primer esfuerzo de Dominique Jean Larrey en las Guerras Napoleónicas (Illesca, 2006). Un elemento fundamental se refiere al personal de salud, quien realiza este tipo de clasificación, debido a que parte de las características propias de la cultura y el sistema que aplique cada institución; tal labor tiene un importante peso en el flujo de usuarios, así como en la clasificación de estos.

Al respecto, investigaciones como la de Lopera, García, Madrigal y Forero (2010), corroboran la problemática en la atención en los servicios de urgencias, desde tres perspectivas:

En la atención de urgencias hay un desencuentro entre tres miradas: la primera es la de los participantes, que la consideran inhumana; la segunda es la del sistema de salud, que la considera un conjunto de actividades que garantizan una atención oportuna y de calidad; y la tercera es la que muestran otras investigaciones que han encontrado barreras de acceso para ser admitido y para obtener información (p.64).

De acuerdo con la cita, los sistemas de clasificación del riesgo (sobre la vida del usuario) buscan mejorar o agilizar la atención en estos servicios y, por consiguiente, la percepción del usuario en cuanto a la atención brindada. Dichos sistemas, que priorizan la atención en los servicios de urgencias, se aplican en diferentes países como Australia, Reino Unido, Canadá y Estados Unidos, donde además se ha desarrollado protocolos propios que orientan la clasificación de los pacientes justo cuando llegan al centro de atención.

Los sistemas más reconocidos llevan el nombre del país donde han sido desarrollados, de esta manera,

los protocolos más utilizados para la clasificación de riesgo en los servicios de urgencia/ emergencia, en nivel mundial, son el Australian Triage Scale (ATSC), Canadian Triage Acuity Scale (CTASC), Emergency Severity Index (ESIC) y el Manchester Triage System (MTSC). Todas esas escalas organizan el servicio en cinco niveles de prioridad (Pinto, Oliveira y Machado, 2012, s.p.).

Respecto de lo anterior, cabe destacar que estos son sistemas de cinco categorías de riesgo para la vida, las cuales priorizan la atención de las personas que llegan a solicitarla en las salas de urgencias hospitalarias. A pesar de su utilidad y relevancia, en muchos países se carece de un sistema estructurado y, además, se desconoce su efectividad, así como su impacto en cuanto a la disminución de los tiempos de espera y, por ende, de la mortalidad. Ante esta panorámica y el volumen impresionante de investigaciones asociadas a los sistemas de clasificación de usuarios en los servicios de urgencias hospitalarias, se presenta este documento, el cual responde 
al proyecto de investigación $\mathrm{N}^{\mathrm{o}}$.421-B1-235, titulado Estrategias para la atención y clasificación de usuarios(as) en los servicios de urgencias a nivel hospitalario, el cual ha recibido la aprobación de la Vicerrectoría de Investigación de la Universidad de Costa Rica.

La relevancia del proyecto radica en su afán de encontrar evidencia sobre los sistemas de clasificación de usuarios en los servicios de urgencias, los profesionales que participan, así como de la capacitación que deben tener para implementarlos, de manera que se agilice la atención de aquellas personas, quienes acuden con patologías que comprometen seriamente su vida.

Esta investigación se realizó mediante la metodología de práctica basada en evidencia y respondió a la siguiente pregunta PICO: ¿En personas que asisten a los servicios de urgencias, el aplicar un sistema estructurado de clasificación en contraste con la atención aleatoria por llegada, reduce la mortalidad y los tiempos de espera?

\section{MATERIALES Y MÉTODO}

La investigación se realizó mediante la metodología de práctica clínica basada en evidencia, la cual incluye cinco etapas: elaboración de una pregunta de investigación (acrónimo PICO), búsqueda de literatura, lectura crítica de la información, aplicación de los resultados encontrados y evaluación.

En el caso de la pregunta formulada, se efectuó una búsqueda de la literatura nacional e internacional, publicada en los últimos 15 años, la cual se extrajo de las bases de datos, google académico, PubMed, Cochrane library y EBSCO. El proceso para recolectar información se realizó a lo largo del 2012 y 2013.

Dentro de los criterios de inclusión se consideró que los estudios no sobrepasaran los 15 años de publicación y que el estudio fuera en triage hospitalario y en personas adultas. Además se incluyó estudios que observaron la efectividad de los sistemas de triage, el personal que lo aplica, sistemas de triage, tiempos de espera, calidad del servicio.

Después de varías búsquedas, se concluye que PubMed y google académico contienen los artículos que se identificaron en Cochrane Library, EBSCO, razón por la que en la figura 1 se describe la forma en que se estructuró la búsqueda en dichas bases. 


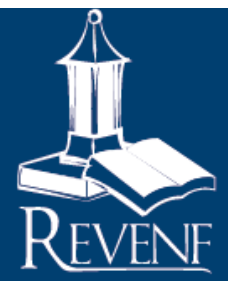

Revista Electrónica Enfermeria Actual en costa Rica

\section{Figura 1}

Proceso de búsqueda y extracción de documentos de internet

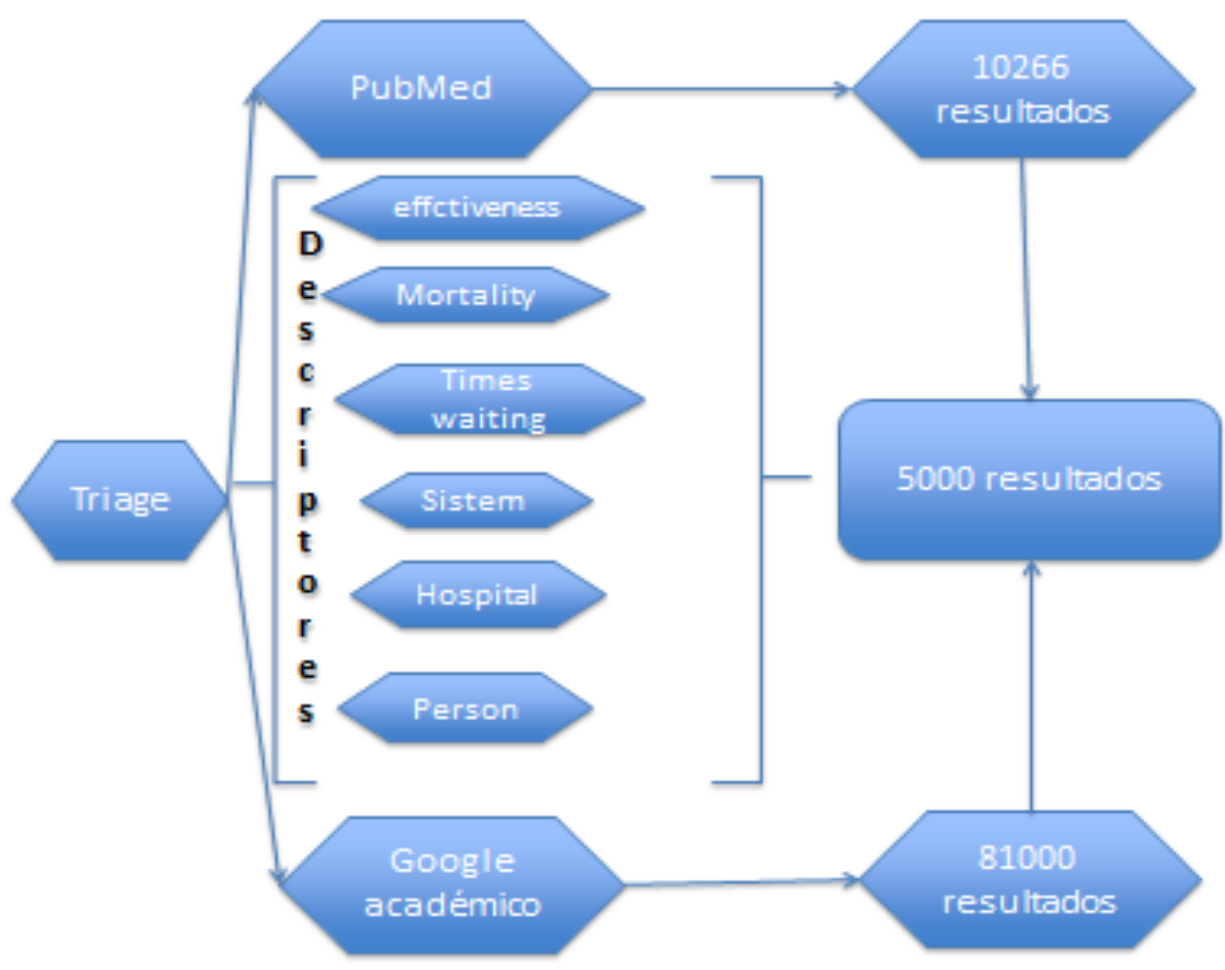

Esta búsqueda se realizó en idioma español e inglés: a los documentos extraídos se les aplicó el proceso de lectura crítica. A continuación en la figura 2 se presenta desarrollado. 


\section{Figura 2}

\section{Proceso de selección de artículos}

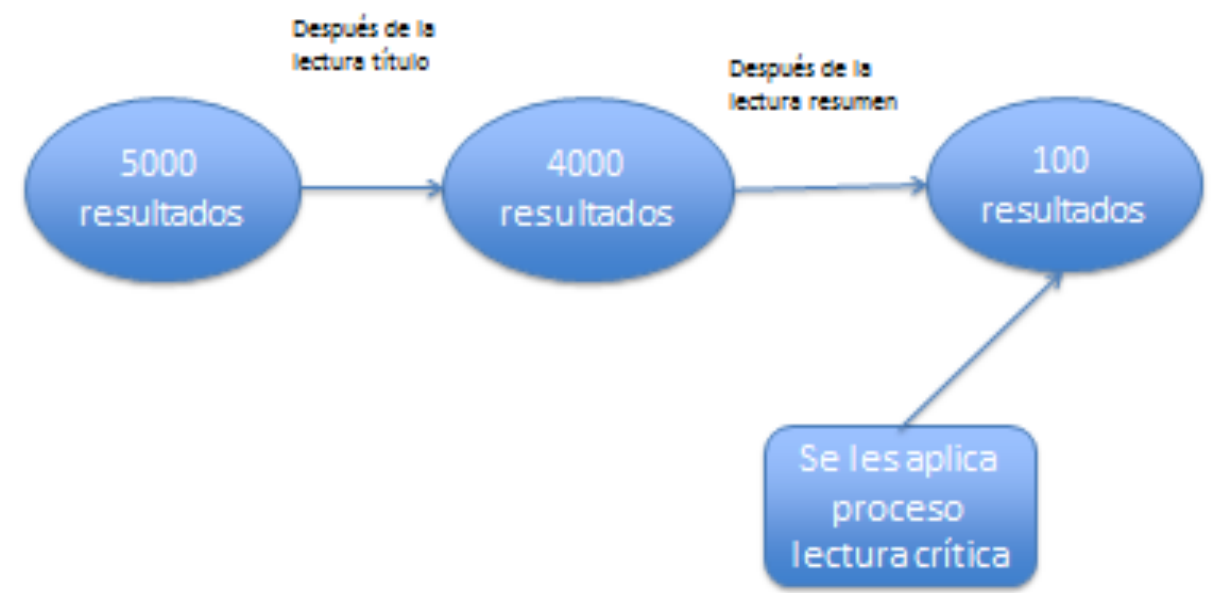

Los documentos que superaron este proceso son los que se utilizaron y se presentan como resultado, cabe destacar que el proceso de aplicación y evaluación de los resultados culminará al presentar los resultados a las personas encargadas de la toma de decisiones a nivel institucional.

\section{Consideraciones éticas}

Para cada material usado, se respetó los derechos de autor y, a través del texto, se incorporó las citas bibliográficas correspondientes.

\section{RESULTADOS}

Después de un proceso de búsqueda y revisión de la literatura, se destaca la gran heterogeneidad de la literatura identificada, además que su calidad es mala, puesto que, en su mayoría, se trata de estudios descriptivos y/o opinión de expertos, sustentados en las características de los sistemas de clasificación de usuarios en los servicios de urgencias. De lo anterior se colige que existen pocos puntos estadísticos de comparación o síntesis de la literatura identificada, por lo que los resultados se presentan de la siguiente manera:

Acerca de la saturación de los servicios de urgencias

En cuanto a este punto, es importante mencionar que toda la literatura revisada coincide en que hay saturación en las salas de urgencias a nivel hospitalario, así como en que los sistemas de triage ofrecen una respuesta a ese problema.

Respecto del tema, existe una demanda que excede las capacidades de las salas de urgencias a nivel hospitalario, en diferentes latitudes a nivel mundial, un fenómeno que no es nuevo, lo cual es constatado por Miró, De Dios, Antonio, Sánchez, Borras y Millá (1999), quien afirma que los servicios de urgencias son un 
vértice en la pirámide asistencial, en los que son atendidas aquellas personas que vienen de otros niveles así como los que llegan por propia iniciativa, lo cual desemboca en un colapso del servicio. Sobre esta temática, Zaragoza, Calvo, Saad, Morán, San José y Hernández (2009) indican que el uso de estos servicios ha aumentado significativamente en los últimos años, sobre todo en los países desarrollados.

En esta misma línea Álvarez, Gorostidi, Rodríguez, Antuña y Alonso (1998) refieren que los sistemas de triage responden a un desmedido aumento en la demanda de atención en ciertos horarios de los servicios de urgencias, cuyo fin es ordenar las necesidades reales de asistencia y mejorar los tiempos de espera, ambas medidas de control de la calidad del servicio prestado en estas instancias hospitalarias.

En otros estudios más recientes, como el de Mendoza y Elguero, se afirma que "el proceso de clasificación de los pacientes que acuden a los servicios de urgencias hospitalarios se ha convertido en una necesidad debido a la gran demanda de atención y a la saturación que, en ocasiones, sufren dichos servicios" (2011, p.94)

Finalmente, tras revisar la saturación en los servicios de urgencias hospitalarios, Flores confirma que "la saturación de los servicios de urgencias hospitalarios (SUH) es un problema global que afecta a millones de pacientes cada día" (2011, p.59).

\section{Sistemas de clasificación}

Sobre los sistemas de clasificación de usuarios, Candela, Cámara, Valenzuela, Porcel y Romero-Nieva (2006), revisan la definición de dos palabras fundamentales, "clasificar" que la definen como categorizar y "triar", cuyo origen histórico, la remite a clasificar víctimas o personas que requieren atención médica, por ende, ambos conceptos son similares.

Por otro lado, López, Montiel y Lincona (2006) abordan el tema de la evolución histórica de los sistemas de TRIAGE, lo cual coincide con lo mencionado en párrafos anteriores respecto de que surgen con la guerra en el ejército Napoleónico, y se retoman en la Primera y Segunda Guerra Mundial, hasta perfeccionarse. Esta experiencia se aplica a la atención prehospitalaria y luego se inicia su aplicación en los niveles hospitalarios, alrededor de 1990.

$\mathrm{Al}$ igual que dichos autores, Illesca (2006) revisa la historia y menciona las siguientes etapas en el proceso de triage: triage de campo, triage en las salas de urgencias y Triage hospitalario por áreas. En el nivel hospitalario Illesca identifica el sistema cronológico por llegada (en desuso), sistema de lotería (no se utiliza), sistema por criterio (inaceptable por la subjetividad, según el autor ), sistema de puntuación de la severidad de las lesiones (poco práctico) y sistema de servicio de espera. De igual forma, el autor determina tres modelos en la aplicación prehospitalaria: índice de triage, índice de trauma y el sistema START, el más aplicado de los tres.

Más recientemente, Mendoza y Elguero (2011) mencionan que existen cinco modelos de triage en el mundo: "escala de triage australiano(ATS), triage del Departamento de Urgencias de Canadá (CTAS), sistema de triage de Manchester (MTS), índice de gravedad de urgencia (ESI) y el modelo de triage andorrano" (p.94). 
Estos modelos de clasificación se estructuran a partir de cinco niveles de clasificación de usuarios, en los que el nivel 1 se refiere a pacientes con riesgo vital inmediato y el 5, a consultas no urgentes.

Para finalizar, en relación con el tema, Cornejo, Arnáiz y De los Santos concluyen que

el "triage" se convierte en un instrumento valioso de ayuda a la gestión de la asistencia del servicio de urgencias, el cual colabora en la eficiencia del servicio y aporta un orden justo en la asistencia, basado en la urgencia/gravedad de los pacientes. Al margen de disminuir el riesgo de los pacientes, el "triage" aumenta la satisfacción de los usuarios y de los profesionales y racionaliza el consumo de recursos colaborando en la mejora de la calidad global del servicio (2008, s.p).

Personal que realiza el triage

La literatura menciona que a nivel hospitalario existen dos profesionales en salud encargados de la clasificación, los médicos y las enfermeras, acerca de lo que Parrilla, Cárdenas, Vargas, Parrilla, Días y Cárdenas (2003) consideran que no hay diferencia en la clasificación realizada por el profesional de enfermería y la efectuada por el médico; por otra parte, además hacen hincapié en que muchos de las personas que asisten a estos servicios, actúan por iniciativa propia y con problemas de salud de poca importancia que pudieron ser resueltos en otro nivel de salud.

Como parte de la evidencia respecto de la ejecución del triage, por parte de los profesionales de enfermería entrenada, Álvarez (2000) señala que:

1. Se ha conseguido mejor control del flujo de pacientes en el Servicio de Urgencias.

2. El uso del manual de triage y unidad ambulatoria ha supuesto una unificación de criterios, mayor seguridad en el personal de enfermería que los utiliza y mejor asignación de las prioridades al atender pacientes.

3. Se ha incrementado la satisfacción percibida por el paciente y su familia al ser atendido justo cuando llega al Servicio de Urgencias, aparte de que ha disminuido el número de reclamos (figs. 2 y 3: El triage en los servicios de urgencias hospitalarios: papel de la enfermera).

4. Este sistema avanzado de jerarquización de necesidades, realizado por los enfermeros de cada entidad de salud, ahorra dinero, pero también amplía las funciones del profesional de las salas de urgencias hospitalarias (SUH), aunque está capacitado para ellos (Álvarez, 2000, pp.237-238).

Acerca del éxito en el rol del profesional de enfermería en las salas de urgencias en Irlanda, Small refiere que se refleja en "la mejora del servicio prestado, desarrollo profesional, satisfacción expresada por los usuarios, así como en la mejora en los tiempos de consulta y alta" (2010, p.220).

De manera similar FitzGerald, Jelinek, Scott y Frances (2010), revisaron la literatura en torno al triage en el servicio de urgencias y concluyeron que tradicionalmente el proceso de triage es intuitivo, aparte de que se constituye en un elemento de la práctica de enfermería, área desde la que es posible reorganizar las colas y determinar quién debe ser atendido de primero. 
Lo expuesto por dichos autores se ratifica en la investigación de Salmerón, Jiménez, Miró y Sánchez (2011) quienes, tras su estudio, manifiestan que "la conclusión más importante es que la derivación sin visita médica llevada a cabo por el personal de enfermería desde el triage del SUH es efectiva y segura” (p.351). Mortalidad y tiempos de espera

La saturación en los servicios de urgencias tiene dos componentes más, los tiempos de espera y la mortalidad, elementos acerca de los que Guttmann, Schull, Vermeulen, Stukel (2011), concluyen que los tiempos de espera más largos se asocian con un mayor riesgo de muerte a corto plazo; aparte, se refieren a los pacientes que se van sin ser vistos (por los largos tiempos de espera), quienes también tienen un riesgo elevado de sufrir alguna complicación.

En los que respecta a este tema, Flores (2011) también establece un aumento en la mortalidad asociada a mayores tiempos de espera, aunque hay diversas variables que intervienen al respecto.

\section{DISCUSIÓN}

El aumento en la demanda de atención en los servicios de urgencias a nivel hospitalario es un problema que se presenta en todo el mundo, fenómeno originado por diversos factores entre los que se destaca qué entiende el usuario acerca de los conceptos urgencia y gravedad, así como la facilidad para recibir atención en estas instancias hospitalarias.

Otro de los aspectos que se mencionan en la literatura y que importantes relevante es que las salas de urgencias fungen como filtro de entrada al sistema hospitalario y también en lo que se refiere a la atención de especialistas, entre otros, lo cual conlleva una demanda desde los niveles de atención más bajos del sistema de salud y también el uso por iniciativa propia de las personas que ven en el sistema una forma de resolver rápidamente una situación determinada de salud.

Los problemas por la saturación en los servicios de urgencias se han dado desde hace ya más de 15 años, hecho en el que los sistemas de clasificación contribuyen a responder a tal necesidad. Entonces, es claro que en la evolución histórica de los servicios de urgencias, la atención aleatoria ha dejado de ser en sí una estrategia de atención, por tanto, dicha transformación produjo que los sistemas de triage se convirtieran en una alternativa para agilizar la atención y mejorar el estándar de tiempos de espera y mortalidad en centros de salud.

Paralelo a esta problemática, se introduce los tiempos de espera y la mortalidad, variables en las que la saturación influye de manera directa, de modo que los sistemas de triage son fundamentales para mejorar las condiciones en la prestación de servicios a nivel hospitalario. Sin embargo, en la temática de mejora de los tiempos de espera y la mortalidad de los servicios de urgencias hospitalarios, la literatura es clara al afirmar que los profesionales de enfermería son quienes deben encargarse de ejecutar los procesos de clasificación, debido a que, histórica y antropológicamente, cuentan con las características idóneas para aplicar un sistema de triage para mejorar la prestación de ese servicio.

En síntesis, se recalca la divergencia entre los sistemas de clasificación y la atención por llegada a las salas de urgencias hospitalarias, además de que hay resaltar que los sistemas son indispensables para el funcionamiento actual de dichas unidades, debido a que la demanda de los servicios brindados en las salas de urgencias es cada 
vez mayor, no obstante, para obtener la respuesta deseada, el personal de enfermería debe aplicarlos, no sin antes haber recibido la capacitación y entrenamiento exigido, acciones que repercutirán en la mejora de la percepción del servicio, los tiempos de espera y, quizás, en la mortalidad.

A pesar de sus ventajas, se cuestiona el decremento de la mortalidad, puesto que en las salas de urgencias existe gran cantidad de variables que pueden influir en esta, las cuales no estarían directamente asociadas a una atención oportuna, tal es el caso de la disponibilidad de una cama o una sala de cirugía de manera oportuna para tratar la condición del usuario que asiste al servicio de urgencias.

Por último, es claro que la nueva línea de investigación en este sentido versa sobre la efectividad real de estos sistemas, acerca de los que se afirma que apenas están adaptándose al nivel hospitalario, aun cuando nacen de un sistema prehospitalario, de igual modo, los vinculan con características culturales y, si bien existen cinco sistemas de clasificación ampliamente reconocidos a nivel internacional, se desconoce su efectividad real, debido a que esta varía según sea el personal que los aplica, el entrenamiento que tenga, los recursos con que cuenta, entre muchas otras variables.

\section{CONCLUSIONES}

Los sistemas de triage se constituyen en la mejor alternativa para dar respuesta a la saturación que existe actualmente en los servicios de urgencias a nivel hospitalario.

Se concluye que para obtener el efecto deseado en la aplicación de los sistemas de triage, deben ser aplicados por personal debidamente entrenado para tal efecto, entre el que se destaca el profesional de enfermería.

Se requiere más investigación en cuanto a la efectividad de los sistemas hospitalarios de triage, y cómo influye el entrenamiento de las personas que lo aplican.

\section{Agradecimiento}

A la Dra Milena Castro por sus comentarios y aportes para mejorar la presentación de esta investigación.

\section{REFERENCIAS BIBLIOGRAFICAS}

Álvarez, B., Gorostidi, J., Rodríguez, O., Antuña, A. y Alonso, P. (1998). Estudio del triage y tiempos de espera en un servicio de urgencias hospitalario. Emergencias 10 (2).

Álvarez, M. (2000). El triage en los servicios de urgencias hospitalarios: papel de la enfermera. Enfermería Clinica, 11(5), 58-66.

Candela, M., Cámara, S., Valenzuela, A., Porcel A. y Romero, J. (2006). Recepción, acogida y clasificación de pacientes en urgencias: RAC \& TRIAGE. Excelencia Enfermera, 13. 
Cornejo, C., Arnáiz, M. y De los Santos, J. (2008). Triage en un servicio de urgencias hospitalarias. Recuperado de http:/www.enfermeriadeurgencias.com/ciber/septiembre/pagina8.html

FitzGerald, G., Jelinek, G.A., Scott, D. y Frances, M. (2010). Emergency department triage revisited. Emerg Med, 27, 86-92. doi:10.1136/emj.2009.077081

Flores, C. (2011). La saturación de los servicios de urgencias: una llamada a la unidad. Emergencias, 23, 59-64

Guttmann, A., Schull, M., Vermeulen, M. y Stukel, T. (2011). Association between waiting times and short term mortality and hospital admission after departure from emergency department: population based cohort study from Ontario, Canada. BMJ, 1. doi: 10.1136/bmj.d2983.

Illescas, G. (2006). Triage: atención y selección de pacientes. Trauma, 9(2), 48-56.

Lopera, M., García, M., Madrigal, M. y Forero, C. (2010). Dificultades para la atención en los servicios de urgencias: la espera inhumana. Investigación y Educación en Enfermería, 28 (1), 64-72

López, J., Montiel, M. y Lincona, R. (2006). Triage en el servicio de urgencias. Medicina Interna de México, 22(4), 310-318.

Mendoza, G. y Elguero, E. (2011). Sensibilidad del triage clínico en el Servicio de Urgencias Adultos del HRLALM del ISSSTE. Archivos de Medicina de Urgencias de México, 3(3), 93-98.

Miró, O., De Dios, A., Antonio, M., Sanchéz, M., Borras, A. y Millá, J. (1999). Estudio de la mortalidad en un servicio de urgencias de medicina hospitalario: incidencias, causas y consecuencias. Med Clin, 112(18)

Parrilla, F., Cárdenas, D., Vargas, D., Parrilla, E., Días, M. y Cárdenas, A. (2003).Triage en urgencias: Facultativo versus Enfermería. Emergencias, 15, 148-151.

Pinto, D., Oliveira, P. y Machado, T. (2012). Validez predictiva del Protocolo de Clasificación de Riesgo de Manchester: evaluación de la evolución de los pacientes admitidos en un pronto atendimiento. Rev. Latino-Am. Enfermagem, 20(6): [08 pantallas]

Salmerón, J., Jiménez, L., Miró, O. y Sánchez, M. (2011). Análisis de la efectividad y seguridad de la derivación sin visita médica desde el triaje del servicio de urgencias hospitalario por personal de enfermería acreditado utilizando el Programa de Ayuda al Triaje del Sistema Español de Triaje. Emergencias, 23, 346-355

Small, V. (2010). El desarrollo de un rol de práctica avanzada en enfermería de urgencias y emergencias: reflexiones desde la experiencia en Irlanda. Emergencias, 22, 220-225.

Zaragoza, M., Calvo, C., Saad, T., Morán, F.J., San José, S. y Hernández, P. (2009). Evolución de la frecuentación en un servicio de urgencias hospitalario. Emergencias, 21, 339-345. 